\title{
High-Frequency Oscillations and Their Importance in Epilepsy
}

\author{
Ofer Michael Gonen \\ Department of Neurology, Assaf Harofeh Medical Center, Israel \\ *Corresponding author: Ofer M. Gonen, The Neurology Department, Assaf Harofeh Medical Center, Zerifin, 70300, Israel, Tel: +972-8-9779181; Fax: +972-8-9779183; \\ E-mail: ogonen@gmail.com
}

Rec date: Aug 15, 2014, Acc date: Oct 19, 2014, Pub date: Oct 21, 2014

Copyright: $\odot 2014$ Gonen OM. This is an open-access article distributed under the terms of the Creative Commons Attribution License, which permits unrestricted use, distribution, and reproduction in any medium, provided the original author and source are credited.

\begin{abstract}
High-frequency oscillations are changes in electrical potentials in the EEG in frequencies above $80 \mathrm{~Hz}$. In recent years it was discovered that these oscillations have great importance both in physiologic processes and as an expression of epileptogenic areas in the cerebral cortex. The measurement of these oscillations via depth-electrodes during pre-surgical evaluation of epilepsy patients has been proven to be an important tool in improving the ability to delineate exactly the area which should be resected, in case such an operation is feasible. New technical developments enable the acquisition of high-frequency oscillations from scalp electrodes as well, which could have great importance in brain research and care of epilepsy patients in the future.
\end{abstract}

Keywords: High-frequency oscillations; Epilepsy surgery; Intracranial electrodes

\section{Introduction}

Since the pioneering studies of Berger the EEG has been a valuable means of research and diagnosis of neurological diseases [1]. The frequencies which are used in the majority of clinical applications are below $70 \mathrm{~Hz}$. The highest frequency which can be sampled without distortions is half the sampling frequency according to Nyquist's sampling theorem [2]. The sampling rate in routine scalp EEG recordings is between $200-500 \mathrm{~Hz}$, which precludes sampling of signals with a frequency of hundreds of oscillations per second [3].

After preliminary clues of clinically significant high-frequency oscillations (HFOs) in humans [4], HFOs were recorded methodically in rats [5]. Later, microwires that extended beyond the tips of clinical depth electrodes were implanted in the brains of epilepsy patients undergoing invasive monitoring, and the signals in a wide range of frequencies were investigated [3]. It was discovered that the spectrum of frequencies above $80 \mathrm{~Hz}$ includes signals of several kinds. Ripples, which are defined as HFOs with a frequency between $80-200 \mathrm{~Hz}$ exist normally in the hippocampus and in certain parahippocampal structures. These oscillations are considered important for the consolidation of memories [6]. Fast Ripples, between $250 \mathrm{HZ}$ and 600 $\mathrm{Hz}$, were discovered to be associated with epileptogenic cortex [7]. Subsequently, HFOs were also demonstrated in the brains of epileptic patients with macroelectrodes used for invasive monitoring $[8,9]$.

Normal ripples probably result from the summation of synchronous inhibitory post synaptic potentials generated by subsets of interneurons that regulate the discharges of principal cells, whereas fast ripples usually reflect the synchronized firing of abnormally bursting neurons [10].

\section{HFOs in Epilepsy patients}

Approximately one-third of epilepsy patients suffer from drugresistant recurrent seizures, despite appropriate medical treatment with at least two drugs of adequate dosage [11]. Refractory epilepsy may cause progressive damage to brain structures from the seizures themsleves, and may lead to psychological comorbidities, various injuries, and increased mortality (e.g. from suicide, accidents, and sudden unexpected death in epilepsy). Therefore, the burden of refractory epilepsy, both on the patients and on society, is substantial [12].

When drug treatments fail, other treatment options include resective surgery, disconnection procedures (e.g. corpus callosotomies), and various neurostimulation techniques (vagus nerve stimulation, deep brain stimulation, responsive neurostimulation). Some improvement can sometimes be achieved with various diets, most notably the ketogenic diet and modified Atkins diet [12].

The best hope of achieving freedom from seizures in patients with drug-resistant epilepsy is resective epilepsy surgery, if such an operation is feasible [13]. In the presurgical evaluation of such patients sometimes invasive monitoring is used, in which electrodes are inserted intracranially in order to map with high precision the seizure onset zone. Interictal spikes are not ideal biomarkers for the purpose of delineating the seizure onset zone. It seems that there are spikes which indeed derive from epileptogenic tissues, but other spikes are merely propagated, or derive from irritable tissue incapable of generating spontaneous seizures [10].

In studies with microwires inserted into the medial temporal lobes of such patients it was discovered at the turn of the century that ripples exist bilaterally in the hippocampal and entorhinal cortices during non-REM sleep. In contrast, fast ripples were recorded only ipsilaterally to epileptic foci [14]. These findings resembled previous findings in a rodent model of epilepsy, in which it was also demonstrated that HFOs can detect the future development of recurrent seizures [15]. Many more studies revealed a correlation between HFOs and epilepsy, and it was concluded that HFOs, in particular fast ripples, are biomarkers for epileptogenic areas [14].

It is important to note that it is not possible to determine whether HFOs are pathologic or not based on the frequency alone, since there are cases in which fast ripples are recorded from normal cortex [10]. An opposite example is recording ripples $(<200 \mathrm{~Hz})$ from the dentate gyrus, which always implies the existence of epileptogenic tissues. 
Page 2 of 3

Therefore, trying to find criteria in order to decide whether HFOs are pathologic or not is an active area of research.

One way of trying to improve the sensitivity of HFOs as biomarkers of epileptogenic areas is to take into account the existence of interictal spikes in the EEG in addition to HFOs [10]. However, even this combination does not provide a full solution to the problem. On the one hand, it is well known that interictal spikes can exist in perfectly normal individuals. On the other hand, it has been shown that HFOs by themselves can reveal the seizure onset zone independently of the existence of interictal spikes [16]. Moreover, HFOs delineate the seizure onset zone even better than structural abnormalities in magnetic resonance imaging [17].

\section{HFOs in Epilepsy Surgery}

The results mentioned previously suggest that interictal HFOs may have an important role in mapping the critical areas for resection in epilepsy surgery in order to achieve seizure-freedom. In a landmark study, twenty patients who underwent resective epilepsy surgery for medically intractable epilepsy were evaluated by presurgical intracerebral EEG and postsurgical MRI and clinical evaluation. Patients with a good surgical outcome (Engel class 1 \& 2) had a significantly larger proportion of HFO-generating areas removed than patients with a poor outcome (Engel class $3 \& 4$ ). No such difference was demonstrated for spike-generating regions or the seizure onset zone, as determined by the intracranial monitoring [18].

The role of ictal HFOs in epilepsy surgery is less well defined. In one study of six patients with neocortical epilepsy, ictal HFOs properly identified the epileptogenic zone and correlated with surgical outcome [19]. In another study of 41 pediatric patients in whom ictal HFOs were recorded, resection of cortical areas with ictal HFOs led to a greater percentage of seizure-freedom $(82 \%)$ in comparison with incomplete resection of those areas (21\%) [20].

Most of the studies about the success of using interictal HFOs in planning the resection in epilepsy surgery where conducted on limbic structures. In a recent study, fifteen patients with neocortical epilepsy underwent invasive monitoring before surgery, and it was discovered that resection of regions with high rates of HFOs was significantly associated with seizure-free outcome [21].

Lately, the use of intraoperative electrocorticography (ECoG) has expanded in epilepsy surgery. This technique is usually used in order to locate interictal spikes. It is known that resecting the areas from which interictal spikes were recorded improves the surgical outcome [22]. In a recent study ECoG was performed during and after epilepsy surgery in which areas suspected as being epileptogenic were resected. It was discovered that resection of areas showing fast ripples was related to good surgical outcome [23].

\section{The Future of HFOs}

Until recently the only way to record HFOs was to use invasive intracranial electrodes, either depth-electrodes or ECoG. However, a number of studies have shown that HFOs can also be acquired by scalp electrodes. After the feasibility of this approach was demonstrated [24], another study has shown that by recording signals simultaneously from scalp and intracranial electrodes one can see a correlation between the two [25]. In a computerized simulation of the ways in which these signals are generated in the cortex and recorded from the scalp it was demonstrated that the signals from the scalp are indeed reliable indicators of cortical oscillations due to the high signal to noise ratio in the recorded frequencies [26].

A few years ago very high-frequency oscillations (VHFOs) were discovered to exist between the frequencies of $1000-2500 \mathrm{~Hz}$ both ictally and interictally. The recording was accomplished with subdural electrodes at a sampling rate of $10 \mathrm{KHz}$. It appears from the preliminary findings that VHFOs differ from HFOs, and have more restricted distribution, smaller amplitude, and different timing of onset [27]. Further research is needed in order to characterize this new kind of electorphysiological entity and it is difficult to speculate as to the nature of its future clinical applications.

\section{Conclusions}

The EEG is still the most important ancillary test in the diagnosis and management of epilepsy. In cases of drug-resistant epilepsy the best chance of attaining seizure-freedom is resection of the epileptogenic area. HFOs represent a new and promising biomarker which can identify epileptogenic cortical tissues. HFOs have already proven themselves as a tool to improve the surgical outcome in epilepsy surgery. The latest developments in noninvasive recording of HFOs have the potential to revolutionize the whole field of epilepsy diagnosis and treatment.

On one end of the spectrum, patients who already have epilepsy, especially refractory epilepsy, will benefit from a way to better locate noninvasively the epileptogenic areas and to assess their compatibility for surgical resection. On the other end are patients after a cerebral injury, or sometimes after a single seizure. In these patients, it is possible that early detection of the epileptogenic process with HFOs will enable us to intervene before chronic seizures ensue, thus improving the prognosis of these patients [28].

It should be noted that other techniques of improving the mapping of the cortical areas which are responsible for seizure onset are also under development. For example, microseizures have recently been described using invasive monitoring techniques [29]. It is possible that combining HFOs with other sophisticated modalities such as functional MRI, magnetoencephalography, and the new ability to detect microseizures will enable us to discover earlier, and to map with greater precision the epileptogenic process than with current technology [30].

\section{References}

1. Berger H. Uber das Flektrenkephalogram des menschen (1929) Arch Psychiatr Nervenkr 87: 527-570.

2. Shannon CE. Communication in the presence of noise (1949) Proceedings of the IRE 37: 10-21.

3. Zijlmans M, Jiruska P, Zelmann R, Leijten FS, Jefferys JG, et al. (2012) High-frequency oscillations as a new biomarker in epilepsy. Ann Neurol 71: 169-178.

4. Fisher RS, Webber WR, Lesser RP, Arroyo S, Uematsu S (1992) Highfrequency EEG activity at the start of seizures. J Clin Neurophysiol 9: 441-448.

5. Bragin A, Engel J Jr, Wilson CL, Vizentin E, Mathern GW (1999) Electrophysiologic analysis of a chronic seizure model after unilateral hippocampal KA injection. Epilepsia 40: 1210-1221.

6. Axmacher N, Elger CE, Fell J (2008) Ripples in the medial temporal lobe are relevant for human memory consolidation. Brain 131: 1806-1817.

7. Bragin A, Engel J Jr, Wilson CL, Fried I, Buzsáki G (1999) Highfrequency oscillations in human brain. Hippocampus 9: 137-142. 
Citation: Gonen OM (2014) High-Frequency Oscillations and Their Importance in Epilepsy. J Neurol Disord 2: 183. doi: $10.4172 / 2329-6895.1000183$

Page 3 of 3

8. Urrestarazu E, Jirsch JD, LeVan P, Hall J, Avoli M, et al. (2006) Highfrequency intracerebral EEG activity $(100-500 \mathrm{~Hz})$ following interictal spikes. Epilepsia 47: 1465-1476.

9. Urrestarazu E, Chander R, Dubeau F, Gotman J (2007) Interictal highfrequency oscillations $(100-500 \mathrm{~Hz})$ in the intracerebral EEG of epileptic patients. Brain 130: 2354-2366.

10. Engel J Jr, Bragin A, Staba R, Mody I (2009) High-frequency oscillations: what is normal and what is not? Epilepsia 50: 598-604.

11. Kwan P, Brodie MJ (2000) Early identification of refractory epilepsy. N Engl J Med 342: 314-319.

12. Laxer KD, Trinka E, Hirsch LJ, Cendes F, Langfitt J, et al. (2014) The consequences of refractory epilepsy and its treatment. Epilepsy Behav 37C: $59-70$.

13. Schuele SU, Lüders HO (2008) Intractable epilepsy: management and therapeutic alternatives. Lancet Neurol 7: 514-524.

14. Jacobs J, Staba R, Asano E, Otsubo H, Wu JY, et al. (2012) Highfrequency oscillations (HFOs) in clinical epilepsy. Prog Neurobiol 98: 302-315.

15. Bragin A, Wilson CL, Almajano J, Mody I, Engel J Jr (2004) Highfrequency oscillations after status epilepticus: epileptogenesis and seizure genesis. Epilepsia 45: 1017-1023.

16. Jacobs J, LeVan P, Chander R, Hall J, Dubeau F, Gotman J (2008) Interictal high-frequency oscillations $(80-500 \mathrm{~Hz})$ are an indicator on seizure onset areas independent of spikes in the human epileptic brain. Epilepsia 49: 1893-1907.

17. Jacobs J, Levan P, Châtillon CE, Olivier A, Dubeau F, et al. (2009) High frequency oscillations in intracranial EEGs mark epileptogenicity rather than lesion type. Brain 132: 1022-1037.

18. Jacobs J, Zijlmans M, Zelmann R, Chatillon CE, Hall J, et al. (2010) Highfrequency electroencephalographic oscillations correlate with outcome of epilepsy surgery. Ann Neurol 67: 209-220.

19. Modur PN, Zhang S, Vitaz TW (2011) Ictal high-frequency oscillations in neocortical epilepsy: implications for seizure localization and surgical resection. Epilepsia 52: 1792-1801.
20. Fujiwara H, Greiner HM, Lee KH, Holland-Bouley KD, Seo JH, et al. (2012) Resection of ictal high-frequency oscillations leads to favorable surgical outcome in pediatric epilepsy. Epilepsia 53: 1607-1617.

21. Cho JR, Koo DL, Joo EY, Seo DW, Hong SC, et al. (2014) Resection of individually identified high-rate high-frequency oscillations region is associated with favorable outcome in neocortical epilepsy. Epilepsia [epub ahead of print DOI: 10.1111/epi.12808].

22. Stefan H, Hopfengärtner R, Kreiselmeyer G, Weigel D, Rampp S, et al. (2008) Interictal triple ECoG characteristics of temporal lobe epilepsies: An intraoperative ECoG analysis correlated with surgical outcome. Clin Neurophysiol 119: 642-652.

23. van Klink NE, Van't Klooster MA, Zelmann R, Leijten FS, Ferrier CH, et al. (2014) High frequency oscillations in intra-operative electrocorticography before and after epilepsy surgery. Clin Neurophysiol [Epub ahead of print DOI: 10.1016/j.clinph.2014.03.004].

24. Andrade-Valenca LP, Dubeau F, Mari F, Zelmann R, Gotman J (2011) Interictal scalp fast oscillations as a marker of the seizure onset zone. Neurology 77: 524-531.

25. Zelmann R, Lina JM, Schulze-Bonhage A, Gotman J, Jacobs J (2014) Scalp EEG is not a blur: it can see high frequency oscillations although their generators are small. Brain Topogr 27: 683-704.

26. von Ellenrieder N, Beltrachini L, Perucca P, Gotman J (2014) Size of cortical generators of epileptic interictal events and visibility on scalp EEG. Neuroimage 94: 47-54.

27. Usui N, Terada K, Baba K, Matsuda K, Nakamura F, et al. (2010) Very high frequency oscillations (over $1000 \mathrm{~Hz}$ ) in human epilepsy. Clin Neurophysiol 121: 1825-1831.

28. Cendes F, Engel J Jr (2011) Extending applications for high-frequency oscillations: the ripple effect. Neurology 77: 518-519.

29. Stead M, Bower M, Brinkmann BH, Lee K, Marsh WR, et al. (2010) Microseizures and the spatiotemporal scales of human partial epilepsy. Brain 133: 2789-2797.

30. Staba RJ, Stead M, Worrell GA (2014) Electrophysiological biomarkers of epilepsy. Neurotherapeutics 11: 334-346. 